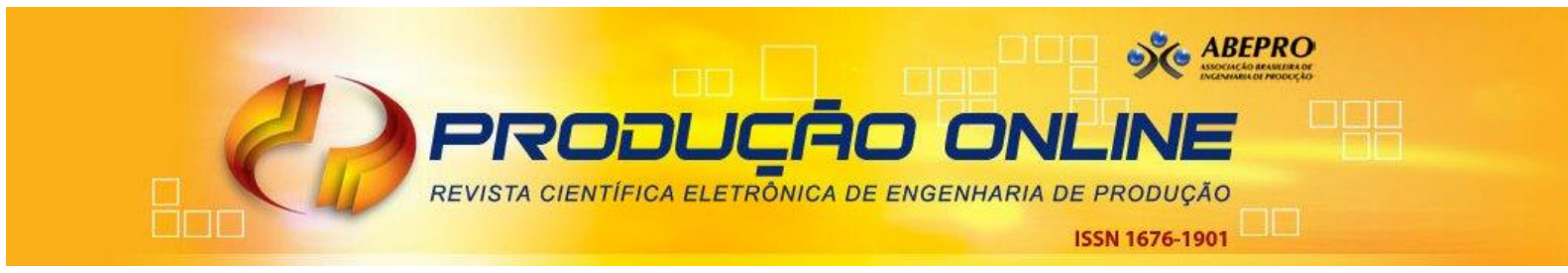

\title{
CONTRIBUIÇÕES DO KANBAN ELETRÔNICO E TRADICIONAL PARA A GERENCIAMENTO DE LEITOS HOSPITALARES
}

\section{CONTRIBUTIONS OF ELECTRONIC AND TRADITIONAL KANBAN TO HOSPITAL BED MANAGEMENT}

\author{
Thainy Genny Esteves Silva* E-mail: thainyesteves@usp.br \\ Mariane Francis Souza Bezerra da Silva** E-mail: maryanefrancis@gmail.com \\ Lara Camila Nery Vieira*** E-mail: laracamilanery@hotmail.com \\ Cristiane Agra Pimentel ${ }^{* * *}$ E-mail: cristianepimentel@ufrb.edu.br \\ Marcel Andreotti Musetti* E-mail: musetti@sc.usp.br \\ * Universidade de São Paulo (USP), São Carlos, SP \\ ** Universidade Ceuma, Recife, PE \\ ***Universidade Federal do Recôncavo da Bahia (UFRB), Feira de Santana, BA
}

\begin{abstract}
Resumo: A falta de leitos resulta em filas de espera e em pacientes em corredores esperando em macas aguardando por leitos. Estes desafios geram a insatisfação nos pacientes e nos profissionais da saúde e podendo causar o aumento de gasto da organização, por conta do tempo elevado de permanência em internações e má gerenciamento dos recursos. O uso do gerenciamento de leito e de conceitos e ferramentas da filosofia Lean Healthcare, como o Kanban que permite melhor controle das demandas e auxilia no aumento da taxa de ocupação das instituições, maior utilização da capacidade disponível, tendo efeito direto no aumento da satisfação dos pacientes. Este artigo pretende apresentar as diferentes características do Kanban tradicional e eletrônico para a gerenciamento de leitos. A metodologia empregada foi a pesquisa exploratória e bibliográfica. Foram utilizados mecanismos de busca como Google Scholar, Web of Science e Scopus, conforme as palavras-chave: lean healthcare, kanban, kanban eletrônico, gerenciamento de leitos, entre outros termos relacionados para uma ampla busca sobre o conteúdo. Com a análise realizada os resultados encontrados foram as contribuições geradas da utilização da ferramenta Kanban eletrônico, que proporciona uma otimização no gerenciamento de leitos através da eficiência no acesso e atualização de dados, aumentando a autonomia dos agentes de saúde e oferecendo atendimentos com qualidade para os pacientes. O estudo contribui para a sociedade acadêmica fornecendo um panorama da utilização da ferramenta Kanban em unidades hospitalares para o gerenciamento de leitos.
\end{abstract}

Palavras-chave: e-Kanban. Kanban tradicional. Gerenciamento de leitos. Contribuições.

Abstract: The lack of beds results in waiting lines and patients in corridors waiting on stretchers waiting for beds. These challenges generate dissatisfaction in patients and health professionals and may cause an increase in the organization's expenses, due to the long stay in hospital and poor management of resources. The use of bed management and concepts and tools of the Lean Healthcare philosophy, such as Kanban, which allows better control of demands and helps to increase the occupancy rate of institutions, greater use of available capacity, having a direct effect on increasing the satisfaction of patients. This article aims to present the different characteristics of traditional and electronic Kanban for bed management. The methodology used was exploratory and bibliographic research. Search engines such as Google Scholar, Web of Science and Scopus were used, according to the keywords: lean healthcare, kanban, electronic kanban, bed management, among other related terms for a broad search on the content. With the analysis performed, the results found were the contributions generated from the use of the electronic Kanban tool, which provides an optimization in the management of beds through efficiency in accessing and updating data, increasing the autonomy of health agents and offering

Revista Produção Online. Florianópolis, SC, v. 21, n. 3, p. 818-836, 2021 
quality care to patients patients. The study contributes to the academic society by providing an overview of the use of the Kanban tool in hospital units for bed management.

Keywords: Electronic Kanban. Traditional Kanban. Bed management. Contributions.

\section{INTRODUÇÃO}

As organizações de saúde possuem desafios diários que afetam a qualidade do atendimento ao paciente diretamente, como a superlotação, consequente de fatores como alta a aleatoriedade da demanda e a variedade no perfil epidemiológico dos pacientes. A falta de leitos resulta em filas de espera mais demoradas e em pacientes em corredores esperando em macas aguardando por leitos. Estes desafios não só geram insatisfação ao paciente e profissionais da saúde como o aumento de gastos da organização (CERDEIRA et al., 2020; ROCHA et al., 2018).

O gerenciamento de leitos possui como um de seus objetivos principais diminuir ou eliminar as situações descritas.

O gerenciamento dos leitos é uma ferramenta que auxilia no aumento da taxa de ocupação de leitos nas instituições, na melhor utilização da capacidade disponível tendo efeito direto no aumento da satisfação dos pacientes. Para isso é necessário detectar todas as atividades que se relacionam com a ocupação de leitos, medi-las, checá-las, e agir sobre o diagnosticado (ALVES, et al., 2019). Aliado ao gerenciamento de leitos, é possível utilizar as práticas de gerenciamento Lean na saúde, conhecida como Lean Healthcare, para oferecer uma assistência segura e de qualidade, conjuntamente com um aumento de produtividade (HENRIQUE; FILHO, 2018).

Em Branco (2017) durante a realização de um estudo de caso descritivo, houve a aplicação do Lean Healthcare, como o Mapeamento do Fluxo de Valor (MFV) e a avaliação dos desperdícios. A autora conseguiu reduzir 517 minutos na utilização do leito por paciente, concluído que práticas do Lean Healthcare possibilita aprimorar a gerenciamento de leitos aumentado a rotatividade dos leitos e diminuindo o tempo de permanência dos pacientes. D'aquino (2017), após verificar a não existência de um modelo integrado de planejamento do gerenciamento de leitos, desenvolveu um quadro para registrar informações, durante uma pesquisa-ação com o objetivo de mensurar, verificar, controlar e administrar os processos de gerenciamento de leitos, bem como os futuros propostos a partir da introdução dos conceitos Lean Healthcare. 
O Kanban é uma das ferramentas do Lean, originada na manufatura, que auxilia no gerenciamento de capacidade e demanda (JUNIOR; FILHO, 2008). Esta ferramenta pode ser adaptada para o ambiente da saúde auxiliando não só no gerenciamento dos suprimentos como em Oliveira (2018), mas também no gerenciamento de leitos. O Kanban é um instrumento gerencial que pode auxiliar na otimização da oferta de leitos, diminuição do valor de permanência total do paciente na organização hospitalar e fluidez no giro de leitos (CERDEIRA et al., 2020).

Identificadas as possibilidades de aplicação e os interessantes resultados obtidos pelo uso do Kanban em gerenciamento de leitos dos hospitais, este artigo pretende apresentar as diferentes características do Kanban tradicional e e-Kanban para a gerenciamento de leitos. Além de trazer as contribuições para a gerenciamento hospitalar e para o paciente.

\section{REFERENCIAL TEÓRICO}

\subsection{Lean Healthcare: ferramentas}

Lean Healthcare (LH) objetiva eliminar os desperdícios e etapas desnecessárias que não agregam valor no atendimento ao paciente, aumentando assim a produtividade e a capacidade operacional dos ambientes de atendimento à saúde (BRITO, 2018).

De acordo com Battaglia e Pinto (2014), o lean na saúde possui seis princípios básicos: criação de valor para o paciente, retirando os desperdícios existentes no processo; prática de melhoria contínua, algo sempre pode ser melhorado; alinhamento estratégico no propósito desejado, todas as atividades realizadas diariamente em prol da saúde necessitam ser relevantes para o objetivo estratégico; respeito às pessoas, onde prezar pela segurança dos pacientes é essencial; gerenciamento visual, na filosofia enxuta os problemas devem ser visíveis para a equipe; padronização com flexibilidade, pois a variabilidade nos sistemas de saúde é um de seus principais problemas. Convém destacar que estes princípios são dependentes uns dos outros e impactam no sucesso da implantação do Lean Healthcare (BATTAGLIA e PINTO, 2014). 
Conforme Graban (2013), os benefícios do Lean Healthcare tratam dos desafios mais comuns do sistema de saúde e sua aplicação se baseia em ações que principalmente levem a redução de oito categorias de desperdícios: falhas, espera, excesso de processamento, potencial humano, transporte, movimentação, estoque e superprodução, a seguir pode ser conferido alguns exemplos desses desperdícios na área da saúde.

- Falhas: realização de exames de maneira indevida, administrar medicamentos errados ou na dosagem errada, conduzir paciente para leito incorreto (BERTANI, 2012);

- Espera: por um serviço ou medicamento vindo de outro setor, médico no aguardo de liberação de uma sala de cirurgia ou colaboradores na espera por desequilíbrio nas suas cargas de trabalho (GRABAN, 2013);

- Excesso de processamento: o preenchimento de um formulário técnico por mais de uma vez, por vários profissionais, o que não colabora para a qualidade e segurança da prestação de serviço médico, constituindo um desperdício (MIN et al., 2019);

- Potencial humano: Funcionários que se sentem superados e deixam de apresentar sugestões para melhorias (GRABAN, 2013);

- Transporte: movimentação intensa de materiais ou equipamentos que deveriam estar no local de seu uso, como prontuários dos pacientes (MIN et al., 2019);

- Movimentação: movimentação excessiva de equipe médica, enfermeiros e demais colaboradores, causada por uma organização não racionalizada dos postos de trabalho (BERTANI, 2012);

- Estoque: a estocagem de materiais concebe uma quantia armazenada, porém sem uso, caracterizando um desperdício. Além disso, medicamentos vencidos precisam ser descartados (MIN et al., 2019);

- Superprodução: execução de monitoramento excessivo em paciente que não necessita de tais cuidados extras, produzir medicamentos na tentativa de antecipar uma demanda (BERTANI, 2012).

A filosofia Lean é composta por uma série de conceitos, princípios e ferramentas. Esses conceitos, princípios e ferramentas são usados para criar e fornecer o maior valor na perspectiva do consumidor, consumindo o mínimo de 
recursos (WERKEMA, 2012). De acordo com Vieira et al. (2020) algumas das principais ferramentas utilizadas na filosofia são:

a) 5S: segundo Min et al. (2019), é uma técnica de organização japonesa que consiste em 5 etapas, referente às iniciais das palavras japonesas: Seiri (utilização), Seiton (organização), Seiso (limpeza), Seiketsu (padronização) e Shitsuke (disciplina). Gerada para promover, por meio da consciência e responsabilidade de todos, disciplina, segurança e produtividade no ambiente de trabalho, evitando assim, situações de desordem, sujeira e perda de tempo;

b) Mapeamento de Fluxo de Valor (MFV) ou Value Stream Mapping (VSM): a ferramenta contribui com o detalhamento e entendimento do processo, é utilizado para diferenciar as fases do processo que trazem valor ou não na visão do cliente/paciente (ZATTAR et al., 2017). De acordo com Brito (2018), a ferramenta ilustra o fluxo físico do paciente, de informações e materiais, do início até o fim, com foco em todo o processo. Nele é elaborado um mapa visual das etapas do processo, com a finalidade de compreendê-lo, assim após a compreensão do estado atual é possível melhorá-lo sem grandes complexidades e mais facilmente (ZATTAR et al., 2017);

c) Gestão Visual: segundo Araújo et al. (2017), é uma ferramenta simples e eficaz que ajuda a todos os indivíduos envolvidos na cadeia hospitalar (aos médicos, gerentes, aos pacientes, entre outros) colaborando através de recursos gráficos e visuais, com informações que tornam o fluxo mais inteligente e menos predisposto a falhas, além de mostrar os resultados operacionais. É utilizada para tornar os problemas perceptíveis, o que facilita as reações e tornam as soluções mais ágeis (GRABAN, 2013);

d) Padronização ou Trabalho padronizado: de acordo com Min et al. (2019) caracteriza-se na padronização dos processos no cumprimento das atividades desenvolvidas pelos profissionais de saúde, por meio do uso de protocolos, estabelecimento de barreiras para evitar o surgimento de erros e alternativas para impossibilitar imprevistos, são ações que garantem a confiabilidade, resiliência e eficiência nos cuidados aos pacientes;

e) Diagrama de espaguete: é uma ferramenta muito simples, que ajuda a estabelecer o layout mais apropriado a partir das observações das distâncias abrangidas na realização de uma atividade ou de um processo definido. 
Baseia-se em um diagrama usado para visualizar ao longo de um fluxo a movimentação de materiais, informações e pessoas. Por meio dessa ferramenta é possível identificar algumas das origens de problemas e onde há perda de tempo na execução das atividades, o nome espaguete surge da semelhança do percurso desenhado (layout) a um prato de macarrão do tipo espaguete, em italiano se escreve spaghetti (FREITAS, 2018);

f) Poka-Yoke: conforme Min et al. (2019) é um termo em japonês que significa barreiras contra erros. A base do poka yoke é prevenir defeitos através da antecipação de sua detecção, e mediante a isso alertar os colaboradores sobre a incidência de um erro. Na saúde, o uso dessas barreiras é importante para evitar que aconteçam erros humanos que comprometam a segurança dos envolvidos, desde o paciente até o colaborador, um exemplo da ferramenta são as cores do painel de gases numa enfermaria;

g) Kanban: Seu uso corresponde na sinalização de requisições ou movimentações de itens através de cartões, sendo comumente utilizada para administrar estoque e gerenciamento da produção (GRABAN, 2013).

\subsection{Kanban e a gerenciamento de leitos}

Termo de origem japonesa, Kanban significa no sentido literal "cartão" ou "sinalização". Seu conceito relaciona-se à utilização de cartões para indicar o andamento do fluxo produtivo nas empresas de fabricação em série. Nesses cartões são indicados os status sobre determinadas tarefas, como "a realizar", "em andamento" ou "concluída". Seu uso permite controlar minuciosamente algumas informações de produção como quando, quanto e o que produzir (SOUSA et al., 2017).

Vale salientar que o kanban como sistema de gestão visual, pode ter seus sinais apresentados de diversas maneiras, não apenas no modelo tradicional, como o cartão, mas também pode ser apresentado em forma de quadro e em outras versões, por exemplo, na forma eletrônica caso do e-Kanban, o primordial é que ele transmita a informação de modo simples e claro (CARVALHO, 2014).

De acordo com Heisler (2012), inicialmente utilizado na indústria como método de controle para auxiliar o planejamento da produção, controle de estoques e fluxos produtivos, essa ferramenta é adaptável em qualquer atividade com produção em 
série, inclusive, nos mais diversos segmentos das organizações de saúde, pois sua utilização contribui no processo de melhoria contínua. Isto significa que nas unidades de saúde, o kanban é um recurso para avaliar a qualidade da assistência, para melhorar os fluxos, taxas de indicadores e rotatividade dos pacientes internados, consistindo assim em uma excelente ferramenta de apoio ao gerenciamento de leitos.

Segundo Sousa et al. (2017), o setor de gerenciamento de leitos é responsável por monitorar o fluxo de pacientes, melhorar o fluxo de atendimento dos pacientes e de internação, além de trabalhar em conjunto com a equipe multiprofissional. De acordo com Valente (2016), a taxa de giro dos leitos relaciona-se a quantidade de vezes que o mesmo leito é utilizado por diferentes pacientes, porém muitas das vezes o paciente fica internado por mais tempo que o necessário, o que leva a redução do número de atendimentos e aumenta a probabilidade de o paciente contrair uma infecção.

A ferramenta kanban no gerenciamento de leitos utiliza a sinalização nas cores verde, amarelo e vermelho, com o intuito de alertar a equipe multiprofissional quanto ao tempo de permanência dos pacientes (CERDEIRA et al., 2020).

Essas cores são estabelecidas com base na tabela do Sistema Único de Saúde (SUS), Sistema de Gerenciamento da Tabela de Procedimentos, Medicamentos e Órteses, Próteses e Materiais Especiais (SIGTAP) e considera o tempo médio de internação, e para que seja possível verificar se a classificação do paciente no kanban está apropriada, deve-se considerar qual o tempo médio de internação (CERDEIRA et al., 2020), conforme demonstrado no quadro 1.

Quadro 1 - Critérios para classificação de cores do kanban no gerenciamento de leitos

\begin{tabular}{|c|c|c|}
\hline \multicolumn{2}{|c|}{ Classificação de cores do Kanban no gerenciamento de leitos } \\
\hline CORES & $\begin{array}{c}\text { PERMANÊNCI } \\
\text { A }\end{array}$ & DEFINIÇÃO \\
\hline
\end{tabular}




\begin{tabular}{|c|c|c|}
\hline Verde & Ideal & $\begin{array}{c}\text { No intervalo de tempo de dias } \\
\text { mínimo de internação, permitido } \\
\text { pela tabela SIGTAP }\end{array}$ \\
\hline Amarelo & Alerta & $\begin{array}{c}\text { Da metade do tempo ou igual ao } \\
\text { total de dias, de acordo com } \\
\text { permitido pela tabela SIGTAP }\end{array}$ \\
\hline Vermelho & Ultrapassada & $\begin{array}{c}\text { Maior que o n.- de dias, } \\
\text { permitido pela tabela SIGTAP }\end{array}$ \\
\hline
\end{tabular}

Fonte: Adaptado de Cerdeira et al. (2020)

Exemplificando os critérios dessa classificação tem-se a internação de um paciente com permanência média de internação de 6 dias. Se o paciente for internado do dia 1 até os 3 dias, o acompanhamento é normal e ele receberá a identificação com sinalização verde. Excedido a metade ou a totalidade de dias de internação (ou seja, de 3 até 6 dias), a cor será amarela, assinalando que a atenção seja maior. Quando o paciente se internar por mais tempo do que a permanência média (isto é, superior a 6 dias), obterá a sinalização na cor vermelha e terá uma atenção especial (CERDEIRA et al., 2020).

Em síntese, o padrão das cores de classificação do kanban enquadra-se na sinalização verde se estiver no intervalo entre o número de dias mínimos permitidos; amarelo se no intervalo entre o período de dias da metade do tempo ou igual ao total de dias; e vermelho se na condição de maior que a quantidade de dias permitidos, levando-se em consideração os valores da tabela SIGTAP do SUS (CERDEIRA et al., 2020).

O uso do kanban possibilita uma comunicação mais assertiva entre os especialistas responsáveis, na obtenção de mais informações sobre o quadro do paciente, conforme as cores sinalizadas (verde, amarelo ou vermelho), que indica o tempo médio de permanência para cada problema, o que permite a equipe médica a tomar decisões, como acelerar exames, responder solicitações de consultas e inclusive agilizar algo emergencial, por conseguinte isso aumenta a taxa de ocupação e a taxa de giro dos internamentos (VALENTE, 2016). 
Por fim, Mattos (2016), relata que por meio do kanban otimiza-se a oferta de leitos, avalia-se a necessidade de transferências dos pacientes, reduz-se o índice de permanência nos hospitais, contribuindo dessa forma com o aumento da rotatividade dos leitos, capacidade resolutiva da assistência hospitalar, o que a torna um fator de desospitalização de forma segura e satisfatória para o paciente, em função disso o kanban se tornou uma excelente ferramenta para gerir e controlar a entrada e saída de pacientes.

\section{METODOLOGIA}

O presente trabalho se enquadra em uma pesquisa exploratória e bibliográfica. Exploratória, pois seu objetivo é identificar e descrever variáveis relacionadas e tornar explícito o conhecimento (GIL, 2002). Se enquadra em bibliográfica, pois se constitui na organização dos estudos existentes acerca do tema selecionado, esclarecendo e resumindo as principais obras, fornecendo informações completas e relevantes da área (VOSGERAU e ROMANOWSKI, 2014). Foram utilizados mecanismos de busca como Google Scholar, Web of Science e Scopus, segundo as palavras-chave: lean healthcare, kanban, e-kanban, gestão de leitos, entre outros termos relacionados para uma ampla busca sobre o conteúdo. A busca realizada nas bases selecionando inicialmente após a leitura: título, resumo e palavras-chave, resultaram em 21 artigos do Google Scholar, 6 artigos da Web of Science e 7 artigos da Scopus. Dentre os 34 artigos, foram selecionados 5 para realizar a análise, o critério utilizado foi por serem estudos que implementaram a ferramenta, que apresentaram resultados e utiliza a versão do Kanban tradicional e eletrônica e em unidades de saúde brasileira.

O fluxo seguido da pesquisa é ilustrado na Figura 1, que se inicia com a formulação da situação a ser estudada, construção do objetivo, leitura e seleção do material, análise e interpretação das informações acerca do material selecionado e, por fim, a redação da pesquisa.

Figura 1 - Desenvolvimento metodológico 


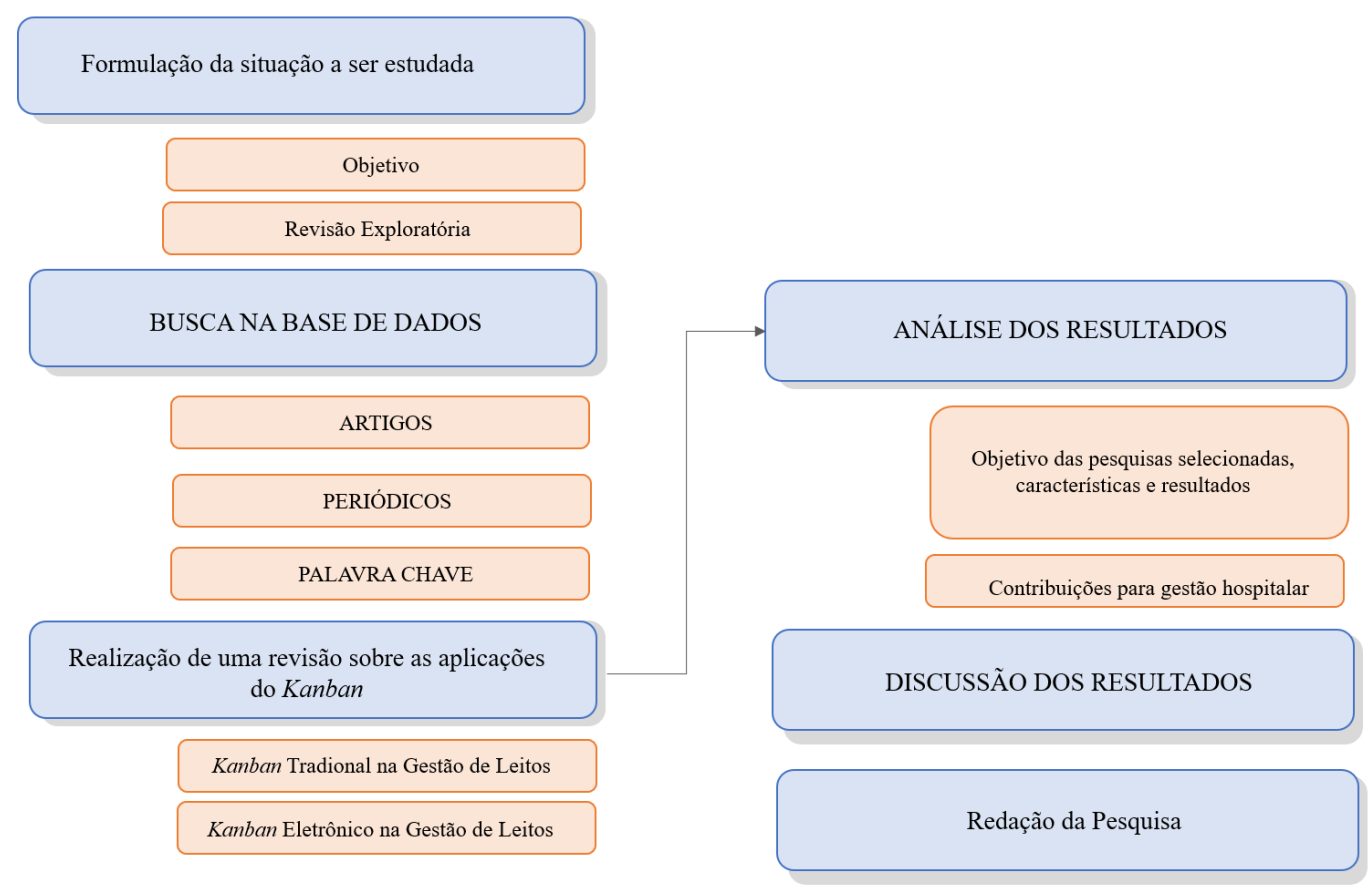

Fonte: elaborado pelo autor (2021)

\section{RESULTADOS E DISCUSSÃO}

Desde o surgimento do Kanban na manufatura sua forma de aplicação vem sofrendo adaptações para que sua utilização se mantenha eficiente em diferentes setores, o "sistema kanban original" possui características apresentadas por Lage Junior e Godinho Filho (2008), as quais serão utilizadas como base referencial para comparação das transformações no sistema kanban no setor da saúde para gerenciamento de leitos. As características do "sistema kanban original" são:

a) Utilização de dois sinalizadores: um sinalizador de ordem de produção e um sinalizador de requisição. O sinalizador de ordem de produção autoriza dá início a atividade, sendo utilizado apenas no setor de processamento. Já o sinalizador de requisição é um mecanismo de controle entre os processos, que informa o que deve ser reposto.

b) A produção é puxada por meio do controle do nível dos estoques finais ou pela programação do último estágio produtivo (FERNANDES e GODINHO FILHO (2007); 
c) A rotina de funcionamento é assegurada de forma descentralizada, por meio do controle visual realizado pelos próprios operários do processo em cada etapa produtiva;

d) Os estoques são limitados em cada estação de trabalho, ou seja, possuem capacidade finita, determinada pelo número de sinalizadores.

Diante das dificuldades em diferentes setores, a busca por melhoria no gerenciamento dos processos é uma prioridade nas instituições, com isso a utilização do Kanban tem se tornado presente em setores distantes da manufatura, ambiente no qual foi concebido. Com isso, as adaptações (diferentes do "original"), surgem para se aproximarem da realidade de cada instituição. Pesquisadores como Vernyi e Vinas (2005), Argenta e Oliveira (2001) expõem a utilização do Kanban com uma modificação principal, de substituição dos sinalizadores físicos por sinalizadores eletrônicos, ou seja, trocar o uso manual proposto no sistema original pelo uso virtual: o sistema e-kanban e/ou kanban eletrônico.

Estudos que apontam a utilização do Kanban tradicional para gerenciamento de leitos e sua versão eletrônica tem se tornado uma realidade nas unidades hospitalares. Com isso será apresentada a análise feita em trabalhos selecionados nas bases de dados mencionadas no 'corpus', que realizaram a implementação da ferramenta Kanban em sua versão tradicional ("original") e/ou eletrônica nos quais será apresentado o objetivo das pesquisas e os resultados acerca da utilização da ferramenta Kanban.

A pesquisa foi realizada por Rocha et al. (2018), um estudo feito durante 4 anos (julho de 2013 a julho de 2017) em um hospital regional em Fortaleza/CE, que possui 300 leitos. Com o objetivo de medir a eficácia do processo de gerenciamento de leitos que utiliza a ferramenta Kanban em sua versão eletrônica, um aplicativo, 'web', que oferece condições de melhorar o fluxo das informações dos pacientes auxiliando na redução do tempo de internação de pacientes hospitalizados. O aplicativo Kanban foi utilizado para gerenciar o tempo de permanência dos leitos, com uma 'interface' gráfica e a distribuição equivalente a real dos leitos da unidade hospitalar. Seu acesso pode ser realizado através de um celular e computadores, e as atualizações feitas pelos usuários são visualizadas em tempo real. A adaptação feita para o modelo 
eletrônico manteve a característica principal do Kanban tradicional, baseando-se principalmente na proposta do Kanban Reativo Descentralizado, feito por Nakamura (1999). A utilização na lógica de cores para os leitos foi mantida, que sinalizam o estágio do processo de alta em que cada paciente se encontra, apresentando assim a necessidade de atenção e para a ação da equipe gestora do leito, conforme a prioridade: vermelho (até 48 horas), amarelo (em até 5 dias) e verde (em até 10 dias). Além disso, a versão eletrônica estratifica todos os pacientes conforme a categoria de atendimento com base em riscos sociais e clínicos, entre outros, e emite relatório diário geral. O sistema também possui uma sinalização automática com informações sobre a demanda para os gestores, e o sistema monitora e analisa a previsão de alta. Com tudo, os autores, Rocha et al. (2018) concluíram que a utilização do Kanban em uma versão eletrônica é eficaz na redução do tempo de internação dos pacientes, pois obtiveram em sua pesquisa que após a intervenção o tempo de internação geral do hospital foi reduzido de 5,6 dias para 4,9 dias e a porcentagem relativa a reclamações relacionadas à disponibilidade de leitos no hospital caiu de $27 \%$ para $0 \%$.

O estudo, realizado por Cecílio et al. (2019) apresenta a visão dos profissionais após a implementação da ferramenta Kanban para atender a superlotação nos serviços de urgência e emergência. O monitoramento foi realizado por dez meses em um hospital municipal, e as observações foram registradas em diários de campo. Tendo como objetivo para implementação do Kanban surgiu a partir da excessiva demanda, com superlotação e reclamações sobre a qualidade da assistência à saúde, com tempo de espera elevados e/ou indisponibilidade de leitos, situações manifestadas pelos pacientes. A lógica do Kanban no estudo foi baseada em dois eixos principais, sendo eles: o trabalho interprofissional para melhorar a qualidade dos diagnósticos e o manejo clínico; e o uso de informações atualizadas em tempo real sobre o paciente e compartilhadas pela equipe, garantindo um fluxo ágil de rotatividade e utilização dos leitos. Após análise dos processos internos, foi possível identificar que o Kanban acelerou ou escalou a especialização e fragmentação da prática dos enfermeiros, recebendo avaliações positivas acerca da incorporação da nova tecnologia. As observações dos enfermeiros que foram apresentadas na pesquisa, diziam conforme Cecílio et al. (2019) que a utilização do Kanban "contribuiu para organizar o fluxo do hospital" e "antes do Kanban não sabíamos o que estava acontecendo com os pacientes, o que fora feito, o que eles estavam esperando. (...) 
o trabalho era fragmentado e desorganizado; a mudança foi muito significativa". Portanto, a pesquisa concluiu que o Kanban causou mudanças essenciais para a prática profissional, que com a adoção relativamente recente do dispositivo não permitiu uma avaliação mais abrangente da transformação que ainda se encontrava em andamento, mas, foi possível identificar pontos positivos.

Lang (2018), apresenta em sua pesquisa realizada na Rede Ebeserh uma proposta de intervenção para difundir as etapas de um modelo de Gerenciamento Assistencial para hospitais universitários, baseados na cogestão e no dispositivo Kanban, e relacionada também o Núcleo Interno de Regulação (NIR) e o painel de indicadores. Para embasamento da proposta de intervenção a autora selecionou 21 materiais sendo eles artigos e livros, dentre eles 7 artigos sobre a utilização da ferramenta Kanban em ambiente hospitalar, 11 sobre a regulação intra-hospitalar de leitos. Segundo Heisler (2012), o Kanban possui como um dos principais objetivos no serviço da saúde avaliar o gerenciamento de leitos no processo de internação hospitalar, considerando a média de permanência de pacientes e a disponibilidade para atender a demanda. Portanto, a pesquisa realizada pode contribuir com o resultado da implementação de um Modelo de Gerenciamento Assistencial para os hospitais universitários da Rede Ebserh, baseado na cogestão e no dispositivo Kanban, proporcionando uma qualificação da eficiência do serviço oferecido e colocando em destaque os hospitais públicos.

Oliveira (2019) traz a pesquisa que trata da elaboração de fluxogramas e protocolos para gerenciamento de leitos no setor de urgência e emergência, e o desenvolvimento de um 'software' para monitoramento gerencial Kanban. A pesquisa foi realizada em um hospital universitário, durante o período de maio de 2018 a maio de 2019. O Kanban em unidades hospitalares, busca qualificar o gerenciamento do cuidado, permitindo que o paciente internado possa ser identificado, e a equipe responsável, o diagnóstico e o tempo de permanência (MASSARO e MASSARO, 2017). A pesquisa destaca os benefícios e desafios para implantação do Kanban, em que definiram como benefícios a otimização dos serviços prestados, para maior rotatividade dos leitos e oferecimento de vagas para atendimentos, a melhoria da qualidade e segurança do paciente e do profissional, com direcionamento do paciente ao recurso adequado. $O$ trabalho ofereceu como resultado protocolos de implantação e implementação da ferramenta Kanban; fluxogramas dos processos; mapeamento 
de conteúdo e como produto final da pesquisa o 'software' para monitoramento da ferramenta Kanban.

O 'software', "CuidarTech Kronos”, desenvolvido para gerenciamento de leitos no setor de urgência e emergência que utiliza a metodologia Kanban apresentado por Oliveira (2019) foi considerado até o momento inédito, desenvolvido conforme as necessidades da unidade. A pesquisa apresenta um potencial de aplicação ao nível local, regional e nacional, por trazer um impacto relevante através das descrições sistematizadas das etapas e recursos necessários para a implantação e implementação do Kanban para o gerenciamento dos leitos no setor de urgência e emergência. No âmbito hospitalar não possui uma metodologia padronizada de implantação do Kanban, tornado o estudo como fonte de embasamento para utilização em outras unidades como norteador.

O trabalho realizado por Petry (2016) traz como principal objetivo analisar a implantação do Kanban, estimando seu grau de implantação e descrever os efeitos acerca dos fatores favoráveis e desfavoráveis. Com a elaboração de modelos lógicos operacionais, causais e teóricos do Kanban, através do estudo feito em 7 dos 12 hospitais participantes da primeira etapa do Programa SOS Emergência. O resultado da análise feita sobre o grau de implantação do sistema Kanban nas unidades trouxe que um hospital tem a ferramenta implantada totalmente, quatro parcialmente implantadas, um insatisfatoriamente e uma não implantada. Foram identificadas contribuições contextuais para a implantação do Kanban, tendo destaque: as atuações do Núcleo Interno de Regulação; do Núcleo de Acesso à Qualidade Hospitalar, da Equipe Horizontal e da direção hospitalar, e a atuação dos apoiadores do Programa SOS Emergências.

Foi apresentado fatores que podem atrapalhar o processo de implantação do Kanban que foi possível identificar, destacam-se: a direção hospitalar com pouco interesse na utilização e benefícios da ferramenta Kanban; o déficit de pessoal nas equipes de condução; a rotatividade das equipes e pouco conhecimento sobre o uso do Kanban, sistemas de controles manuais que gera um atraso no fluxo de informações, fluxos de informação e processo não definidos com clareza e a resistência de alguns profissionais da saúde. Com a análise realizada é possível concluir que os objetivos do Kanban são atingidos proporcionalmente ao grau de implantação e, que, quando totalmente implantado, consegue atingir todos os 
objetivos traçados, proporcionando agilidade na solução de problemas, melhorando as condições de trabalho para atender a demanda constante em uma unidade hospitalar, diminuindo o tempo de permanência do paciente e auxiliar na implantação do gerenciamento da clínica.

Os estudos selecionados para análise acerca da utilização do Kanban em sua versão tradicional ou eletrônica apresentou resultados que contribuem para sociedade como a melhoria na assistência à saúde, menos tempo de espera no pré atendimento e na permanência em internação e a melhora nesses pontos gera também para a unidade hospitalar a diminuição do número de reclamações, redução de custos e aumento da segurança do paciente e do profissional. Com o levantamento das contribuições foi possível identificar que na versão eletrônica da ferramenta Kanban, oferece informações atualizadas em tempo real, compartilhando com equipe e tornando o fluxo ágil dos dados, auxiliando na tomada de decisão e obtendo como resultado o melhor gerenciamento e direcionamento das demandas, além de trazer contribuições para o gerenciamento hospitalar e para o paciente. A contribuiu para a sociedade acadêmica fornecendo um panorama da utilização da ferramenta Kanban em unidades hospitalares para o gerenciamento de leitos.

\section{CONCLUSÃO}

Em virtude dos trabalhos que vêm sendo realizados em unidades hospitalares nas quais vivenciam desafios constantes para atender a alta demanda e proporcionar a qualidade em sua assistência e garantir a segurança dos pacientes. $O$ elevado tempo de permanência pode ocasionar problemas nas unidades, afetando o nível de qualidade do atendimento ao paciente, as condições de trabalho aos profissionais e a limitação de recursos. A utilização do Kanban em sua versão tradicional já apresenta melhorias quando implementado para a gerenciamento de leitos, por auxiliar na organização das demandas, porém, por se tratar de um ambiente que o fluxo de pessoas e informações acontece de forma contínua o uso de quadros e papeis acaba se torando um limitador para a eficiência e com isso temos a versão eletrônica que vem sendo utilizada para aumentar a agilidade no fluxo de informações e diminuir as falhas em relação à perda de dados. 
Com tudo, o presente trabalho apresentou pesquisas realizadas de implementação da ferramenta Kanban em unidades hospitalares para a gerenciamento leitos, nos quais foi possível concluir que a utilização da versão eletrônica do Kanban proporciona uma redução no tempo de permanência dos pacientes, ocasiona maior organização no fluxo de informações, aumenta a autonomia dos agentes de saúde por terem acesso rápido às informações, gerando também redução de custos, eliminação de desperdícios e oferecendo um serviço de qualidade para os pacientes com a diminuição de reclamações e oferecendo confiança e suporte aos funcionários que executam os processos.

\section{AGRADECIMENTOS}

Os autores agradecem a CAPES (Coordenação de Aperfeiçoamento de Pessoal de Nível Superior) para apoio financeiro no âmbito da bolsa de pesquisa de mestrado (2020/88887.498401). A UFRB (Universidade Federal do Recôncavo da Bahia) para apoio financeiro no âmbito da bolsa de iniciação científica.

\section{REFERÊNCIAS}

ALVES, Aline Luzia de Oliveira; AMARAL, Deidiane do; ZANIN, Jeferson; ALVES, Heloísa. Importância da gestão de leitos para qualidade do atendimento nas instituições de saúde. 2019. Disponível em: http://www.hsan.com.br/wp-content/uploads/2019/11/AImport\%C3\%A2ncia-da-Gest\%C3\%A3o-de-Leitos-Autores-Heloisa-Deidiane.pdf. Acesso em 22 abr. 2021.

ARAÚJO, Jefferson Jesus de et al. Aplicação do Lean Healthcare em um hospital público em Petrolina-PE: melhoria de eficiência a partir da eliminação de desperdícios. In: ENCONTRO NACIONAL DE ENGENHARIA DE PRODUÇÃO, 37., 2017. [Anais...]. p. 1-17. Disponível em: http://www.abepro.org.br/biblioteca/TN STP 238384 33794.pdf. Acesso em: 21 abr. 2021.

ARGENTA, Caio Eduardo Barbosa; OLIVEIRA, L. R. Análise do Sistema KANBAN para gerenciamento da produção com auxílio de elementos de tecnologia da informação. In: ENCONTRO NACIONAL DE ENGENHARIA DE PRODUÇÃO, 21., 2001, Salvador. [Anais...]. Salvador, 2001.

BATTAGLIA, Flávio; PINTO, Carlos Frederico. Aplicando Lean na saúde. Lean Institute Brasil. São Paulo, 2014. Disponível em: https://www.lean.org.br/artigos/262/aplicando-leanna-saude.aspx. Acesso em: 28 abr. 2021.

BERTANI, Thiago Moreno. Lean healthcare: recomendações para implantações dos conceitos de produção enxuta em ambientes hospitalares. 166f. Dissertação (Mestrado) Universidade de São Paulo, São Paulo, 2012. 
BRANCO, Ângela Soares da Cunha Castello. Utilização dos conceitos Lean Healthcare na otimização da gestão de leitos de internação hospitalar. 72f. Dissertação (Pós Graduação em Ciências Aplicadas à Saúde do Adulto) - Faculdade de Medicina, Universidade Federal de Minas Gerais, Belo Horizonte, 2017.

BRITO, Melissa Prado de. Aplicação de técnicas de gestão avançada Lean Helthcare para otimizar o fluxo de pacientes do pronto-socorro de um hospital universitário público de Belo Horizonte. Belo Horizonte: UFMG, 2018. 90p. Dissertação (Mestrado) Programa de Pós-Graduação em Ciências Aplicadas à Saúde do Adulto, Universidade Federal de Minas Gerais, Belo Horizonte, 2018. Disponível em: http://hdl.handle.net/1843/BUOS-BCDPTM. Acesso em: 20 abr. 2021.

CARVALHO, Vítor Hugo de Pina Soares. Implementação de um sistema Kanban na indústria metalomecânica. Aveiro: UA, 2014. 66 p. Dissertação (Mestrado) - Departamento de Economia, Gestão e Engenharia Industrial, Universidade de Aveiro, Aveiro, 2014

CECÍLIO, Luiz Carlos de Oliveira; REIS, Ademar Arthur Chioro dos; ANDREAZZA, Rosemarie; SPEDO, Sandra Maria; CRUZ, Nelma Lourenço de Matos; BARROS, Luciana Soares de; CARAPINEHIRO, Graça; CORREIA, Tiago e SCHVEITZER, Mariana Cabral. Nurses in the Kanban: are there news meanings of professional practice in innovative tools for hospital care management? Ciência \& saúde coletiva, v. 25, p. 283-292, 2019. https://doi.org/10.1590/1413-81232020251.28362019

CERDEIRA, Ana Karina Lima Alves; DE PAIVA, Kairon Ramon Sabino; QUEIROZ, Luis Antônio; RODRIGUES, Valdira Gonzaga. Metodologia kanban como estratégia na gestão de leitos no hospital universitário professor edgard santos hupes. Revista Brasileira De Inovação Tecnológica em Saúde, v. 9, n. 3, 2019. 2020. Disponível em: https://periodicos.ufrn.br/reb/issue/view/976. Acesso em: 22 abr. 2021

D'AQUINO, Sílvio Fernandes. Proposta de modelo de referência para o processo de gestão de leitos hospitalares. Dissertação (mestrado) - Universidade Federal de Santa Catarina, Centro Tecnológico, Programa de Pós-Graduação em Engenharia de Produção, Florianópolis, 2017. Disponível em:

https://repositorio.ufsc.br/xmlui/handle/123456789/183604. Acesso em: 22 abr. 2021.

FERNANDES, Flávio Cesar Faria; GODINHO FILHO, Moacir. Sistemas de coordenação de ordens: revisão, classificação, funcionamento e aplicabilidade. Gestão \& Produção, v. 14, n. 2, p. 337-352, 2007. https://doi.org/10.1590/S0104-530X2007000200011

FREITAS, Eder. Diagrama spaghetti: espaguete. Blog Engenharia de Produção, 2018. Disponível em:

https://engenhariadeproducaoindustrial.blogspot.com/search?q=diagrama+de+espaguete. Acesso em: 21 abr. 2021.

GIL, Antonio Carlos. Como elaborar projetos de pesquisa. 4. ed. São Paulo: Atlas, 2002.

GRABAN, Mark. Hospitais lean: melhorando a qualidade, a segurança dos pacientes e o envolvimento dos funcionários. 2 ed. Porto Alegre: Bookman, 2013.

HEISLER, Paulo Alexandre. Aplicação da metodologia Kanban como ferramenta adaptada para gestão de "leitos" na emergência. Porto Alegre: ICICT, FIOCRUZ, 2012. 25 p. Trabalho de Conclusão de Curso (Especialização em Informação Científica e Tecnológica em Saúde) - Instituto de Comunicação e Informação Científica e Tecnológica 
em Saúde, Fundação Oswaldo Cruz, Porto Alegre, 2012. Disponível em: http://www.arca.fiocruz.br/handle/icict/6505. Acesso em: 22 abr. 2021.

HENRIQUE, Daniel Barberato; FILHO, Moacir Godinho. A systematic literature review of empirical research in Lean and Six Sigma in healthcare. Total Quality Management \& Business Excellence, v. 31, n. 3, p. 429 - 449. 2018.

https://doi.org/10.1080/14783363.2018.1429259

HEISLER, Paulo Alexandre. Aplicação da Metodologia Kanban como ferramenta adaptada para gestão de leitos na emergência. Rio de Janeiro:Fundação Oswaldo Cruz; 2012.

LAGE JUNIOR, Muris Lage; FILHO, Moacir Godinho. Adaptações ao sistema kanban: revisão, classificação, análise e avaliação. Revista Gestão \& Produção, São Carlos, v. 15, n. 1, p. 173-188, jan-abr. 2008. https://doi.org/10.1590/S0104-530X2008000100015

LAGE JUNIOR, Muris; GODINHO FILHO, Moacir. Adaptações ao sistema kanban: revisão, classificação, análise e avaliação. Gestão \& Produção, v. 15, n. 1, p. 173-188. 2008. https://doi.org/10.1590/S0104-530X2008000100015

LANG, Cláudia Emília. Modelo de gestão assistencial para a Rede EBSERH baseado na cogestão, núcleo interno de regulação, Kanban e painel de indicadores. 2018. Dissertação (Mestrado) - Universidade Federal do Rio Grande do Norte - UFRN, Brasil.

MASSARO, Isis Aparecida Cunácia; MASSARO, Altair. O uso do KANBAN na gestão do cuidado: superando limites. Revista de Administração em Saúde, v. 17, n. 66, 2017. https://doi.org/10.23973/ras.66.14

MATTOS, Corina Maria. Aplicação do Kanban ao processo de internação de uma unidade hospitalar pediátrica. Niterói: UFF, 2016. 108 f. Dissertações (Mestrado profissional em Enfermagem Assistencial) - Escola de Enfermagem Aurora de Afonso Costa, Universidade Federal Fluminense, Niterói, RJ, 2016. Disponível em:

https://app.uff.br/riuff/handle/1/3060. Acesso em: 20 abr. 2021.

MIN, Li Li; SARANTOPOULOS, Alice; SPAGNOL, Gabriela Salim; CALADO, Robisom. D. 0 que é esse tal de Lean Healthcare, 2019. https://doi.org/10.22409/leanhealthcare

NAKAMURA, Takahashi K. Reacting JIT ordering systems to the unstable changes in demand. Int J Prod Res, v. 37, p. 2293-313, 1993. https://doi.org/10.1080/002075499190770

OLIVEIRA, Renata Soares de. Kanban e curva ABC: ferramentas de gestão estratégica aplicáveis a unidades hospitalares de abastecimento farmacêutico. 2018. 55 f. Monografia (Graduação em Farmácia) - Escola de Farmácia, Universidade Federal de Ouro Preto, Ouro Preto, 2018.

OLIVEIRA, Izabella Soares de. Gerenciamento de leitos no setor de urgência e emergência. 2018. Dissertação (Mestrado) - Universidade Federal do Espírito Santo, Brasil.

PETRY, Débora. Análise de implantação do Kanban em Hospitais do Programa SOS

Emergências. 2016, Universidade Federal da Bahia, Brasil.

ROCHA, Hermano Alexandre Lima; SANTOS, Ana Kelly Lima Da Cruz; ALCÂNTARA, Antônia Celia De Castro; LIMA, Carmen Sulinete Suliano Da Costa; ROCHA, Sabrina Gabriele Maia Oliveira, CARDOSO, Roberto Melo, CREMONIN Jr, Jair Rodrigues Cremonin. 
Bed management team with Kanban web-based application. International Journal for Quality in Health Care, 2018, v. 30, n.9, pp. 708-714. https://doi.org/10.1093/intahc/mzy108.

RODRIGUES, Valdira Gonzaga. Metodologia kanban como estratégia na gestão de leitos no hospital universitário professor edgard santos hupes. Revista Brasileira De Inovação Tecnológica em Saúde, v. 9, n. 3, 2019. 2020. Disponível em: https://periodicos.ufrn.br/reb/issue/view/976. Acesso em: 22 abr. 2021.

SOUSA, Paulo Renato de; MURICY, Marinês da Silva; SIMEÃO, Érika Pimentel; LIMA, Edvaldo Silva; BRAGA, Bruna Catão. Gestão do Fluxo de Pacientes em Internações Relacionadas ao Pronto Socorro: aplicação da Metodologia Kanban. Revista de Inovação Hospitalar e Inovação em Saúde, Belo Horizonte, v. 14, n. 1, p. 1-18. 2017. https://doi.org/10.21450/rahis.v14i1.3239

VALENTE, Rodrigo. Programa de gestão de leitos é implantado no Hospital de Clínicas de Curitiba. Empresa Brasileira de Serviços Hospitalares - EBSERH, 2016. Disponível em: http://www2.ebserh.gov.br/web/portal-ebserh/detalhes-das-noticias/lasset publisher/7d2qZuJcLDFo/content/id/1413965/2017-05-programa-de-gestao-de-leitose-implantado-no-hospital-de-clinicas-de-curitiba. Acesso em: 25 abr. 2021.

VERNYI, Bruce; VINAS, Tonya. Easing into e-kanban. Industry week-cleveland ohio, v. 254, n. 12, p. 32, 2005.

VIEIRA, Lara Camila Nery; MENEZES, Michelle de Oliveira; PIMENTEL, Cristiane Agra; JUVENTINO, Grace Kelly Sampaio. Lean healthcare no Brasil: uma revisão bibliométrica. Revista de Gestão em Sistemas de Saúde, São Paulo, v. 9, n. 3, p. 381-405, 2020. https://doi.org/10.5585/rgss.v9i3.16882

VOSS, Chris; TSIKRIKTSIS, Nikos e FROHLICH, Mark. Case research in operations management. International Journal of Operations \& Production Management, 2002, v. 22, n. 2, p. $195-219,2002$. https://doi.org/10.1108/01443570210414329

VOSGERAU, Dilmeire Sant'Anna Ramos; ROMANOWSKI, Joana Paulin. Estudos de revisão: implicações conceituais e metodológicas. Revista diálogo educacional, v. 14, n. 41, p. 165-189. 2014. https://doi.org/10.7213/dialogo.educ.14.041.DS08

WERKEMA, Cristina. Lean Seis Sigma: introdução as ferramentas do Lean Manufacturing. 2 ed. Rio de Janeiro: Elsevier, 2012.

ZATTAR, Izabel Cristina; SILVA, Rosangela Rosa Luciene da; BOSCHETTO, Jessica Werner. Aplicação de ferramentas enxutas na área da saúde: uma revisão da literatura. Journal of Lean Systems, Florianopólis, v. 2, n. 2, p. 68-86, 2017.

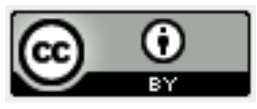

Artigo recebido em: 01/06/2021 e aceito para publicação em: 25/08/2021 DOI: https://doi.org/10.14488/1676-1901.v21i3.4350 